OPEN

SUBJECT AREAS:

MECHANICAL

PROPERTIES

GLASSES

Received

25 July 2014

Accepted

4 November 2014

Published

28 November 2014

Correspondence and requests for materials

should be addressed to

T.D. (thierry.

deschamps@

polytechnique.edu)

\title{
Elastic Moduli of Permanently Densified Silica Glasses
}

\author{
T. Deschamps, J. Margueritat, C. Martinet, A. Mermet \& B. Champagnon
}

ILM, UMR5306 University Lyon 1-CNRS, University of Lyon, 69622 Villeurbanne cedex, France.

Modelling the mechanical response of silica glass is still challenging, due to the lack of knowledge concerning the elastic properties of intermediate states of densification. An extensive Brillouin Light Scattering study on permanently densified silica glasses after cold compression in diamond anvil cell has been carried out, in order to deduce the elastic properties of such glasses and to provide new insights concerning the densification process. From sound velocity measurements, we derive phenomenological laws linking the elastic moduli of silica glass as a function of its densification ratio. The found elastic moduli are in excellent agreement with the sparse data extracted from literature, and we show that they do not depend on the thermodynamic path taken during densification (room temperature or heating). We also demonstrate that the longitudinal sound velocity exhibits an anomalous behavior, displaying a minimum for a densification ratio of $5 \%$, and highlight the fact that this anomaly has to be distinguished from the compressibility anomaly of a-SiO ${ }_{2}$ in the elastic domain.

$\mathrm{t}$ is well known that glassy materials, despite their strong brittleness character, can undergo plastic deformation under high stresses, such as high hydrostatic pressure or indentation ${ }^{1,2}$. Two different mechanisms are involved during plastic deformation: a volume-conservative one, i.e shear flow, and a permanent densification $^{3,4}$. Such densification can vary drastically depending on the atomic packing density, i.e on the free volume of the starting material ${ }^{5,6}$. Greaves et al. have demonstrated in numerous types of glasses, that the Poisson's ratio of an amorphous material (which is related to its atomic packing density), is an excellent criterion to identify its ability to permanently densify. ${ }^{6}$. Unlike metallic glasses or soda-lime silicate glasses, strong oxide glasses such as a$\mathrm{GeO}_{2}$ or a-SiO $\mathrm{S}_{2}$ can densify up to $15 \%$ and $21 \%$ respectively, due to their open, tetrahedrally-connected network. For fused silica, the elastic yield under hydrostatic pressure at ambient temperature is around $9 \mathrm{GPa}^{7}$. Below the elastic yield, the deformation is purely reversible. Above $9 \mathrm{GPa}$, permanent structural changes occur and the retrieved sample is densified. The densification is gradual up to $25 \mathrm{GPa}$ (known as the saturation pressure), a pressure marking the end of the densification process (saturating at 21\%). The elastic yield and the saturation pressure can be reduced if the sample is simultaneously heated during compression or if shear stresses occur'. Between these two pressures (determining the onset of the densification and its saturation), the densification ratio increases continuously ${ }^{7}$. However, despite extensive experimental and simulation studies on $\mathrm{SiO}_{2}$ under high pressure $^{9-30}$, the structural modifications and the elastic properties of such mildly densified glasses are not well understood. This lack of knowledge restricts the modelling of plastic deformation ${ }^{31-35}$.

The aim of this study is to characterize by Brillouin spectroscopy a large set of densified samples after cold compression in order to deduce a relation linking elastic moduli of silica glasses with the densification ratio. From longitudinal sound velocities measurements, we first deduce the longitudinal modulus as a function of the density. Thanks to the Cauchy relation, all the elastic moduli are then calculated, providing phenomenological laws which link a-SiO${ }_{2}$ density with the elastic moduli. These laws display excellent agreement with the sparse data extracted from literature, whatever the thermodynamic path taken during the densification process (room temperature or heating), revealing that the elastic properties only depend on the glass density. Finally, the observed anormal behavior of the longitudinal sound velocity, which shows a minimum at $5 \%$ of densification ratio, is interpreted at the structural scale. The fundamental differences between this anomaly and the well-known compressibility anomaly observed in the elastic regime ${ }^{36}$ are highlighted.

\section{Results}

The diamond anvil cell (DAC) was used to densify Suprasil 300 vitreous $\mathrm{SiO}_{2}$ from Heraeus ( $[\mathrm{OH}<1 \mathrm{ppm}]$ at ambient temperature, applying hydrostatic pressures ${ }^{37}$ (details can be found in the Methods). Seven $\mathrm{SiO}_{2}$ samples were compressed in the DAC at different permanent densification ratios $\Delta \rho / \rho$, depending on the pressure 
Table 1 Maximum pressure reached in the DAC and densification ratio of the recovered samples. Compression was carried out at $T_{\text {amb }}$ with a holding time of $24 \mathrm{~h}$ at $\mathrm{P}_{\text {max }}$. The values of the elastic moduli of the compressed samples are also displayed

$\begin{array}{lcccccrrr}\mathrm{P}_{\max }(\mathrm{GPa}) & 0 & 9.8 & 12.0 & 13.6 & 14.6 & 15.5 & 18.1 & 26.2 \\ \Delta \rho / \rho(\%) & 0 & 1.4 & 4.4 & 8.6 & 11.7 & 14.3 & 19.1 & 21.0 \\ \text { Elastic Moduli } & & & & & & & & \\ \mathrm{L}(\mathrm{GPa}) & 77.3 & 77.3 & 78.4 & 85.6 & 92.2 & 102.8 & 123.2 & 132.2 \\ \mathrm{G}(\mathrm{GPa}) & 30.4 & 30.4 & 30.7 & 32.8 & 34.8 & 38.0 & 44.0 & 46.7 \\ \mathrm{~K}(\mathrm{GPa}) & 36.8 & 36.8 & 37.5 & 41.8 & 45.8 & 52.1 & 64.5 & 69.9 \\ \mathrm{E}(\mathrm{GPa}) & 71.5 & 71.5 & 72.4 & 78.1 & 83.3 & 91.7 & 107.6 & 114.6 \\ v & 0.176 & 0.176 & 0.178 & 0.188 & 0.197 & 0.207 & 0.222 & 0.227\end{array}$

reached. The densification ratios of the compressed samples, which have been already determined in a previous study ${ }^{7}$, are displayed Table 1.

All the recovered samples were then put on a crystalline silicon plate in order to record ex situ the back-scattered Brillouin signal displayed in Fig. 1 (see Methods). In such a configuration, the longitudinal Brillouin shift allows measuring the longitudinal sound velocity using the relation Eq. 1, resulting from the conservation law:

$$
\mathrm{c}_{1}=\frac{\mathrm{v}_{1}}{2 \mathrm{n}} \lambda
$$

where $c_{1}$ is the longitudinal sound wave velocity, $v_{1}$ the Brillouin frequency, $\lambda$ the laser wavelength, and $n$ the refractive index.

Fig. 2a displays the evolution of the longitudinal Brillouin frequency shift as well as the permanent densification ratio as a function of the pressure reached.

Although permanent densification starts from $9 \mathrm{GPa}$, the shift of the Brillouin line, corresponding to the longitudinal acoustic mode, is roughly invariant for $\mathrm{P}_{\max } \leq 12 \mathrm{GPa}$, with a frequency of $32.4 \mathrm{GHz}$. Above $\mathrm{P}_{\max }=12 \mathrm{GPa}$, the Brillouin line shifts monotonously toward higher frequencies and saturates at $40.8 \mathrm{GHz}$ for $\mathrm{P}_{\max }$ $\geq 25 \mathrm{GPa}$. Such variation of the Brillouin shift $v_{1}$ with $P_{\max }$, with an abrupt increase at $12 \mathrm{GPa}$, can be fitted using a Gompertz function rather than a simpler sigmoidal function:

$$
v_{l}=\left(v_{\text {max }}-v_{l o}\right) \exp \left[-\exp \left[-k\left(P_{\max }-P_{c}\right)\right]\right]+v_{l o}
$$

where $v_{10}=32.4 \mathrm{GHz}$ and $v_{\operatorname{lmax}}=40.9 \mathrm{GHz}$ are respectively the Brillouin shift of the non-densified and the fully densified silica, $\mathrm{P}_{\mathrm{c}}=$ $15.0 \mathrm{GPa}$ and $\mathrm{k}=0.546$ are two fitting parameters.

While no variation of the shift of the Brillouin line is observed below $\mathrm{P}_{\max }=12 \mathrm{GPa}$, the elastic yield of silica around $9 \mathrm{GPa}$ has been exceeded, resulting in a permanent densification. The offset between these two pressure values is not an uncertainty as previously shown from Raman measurements on the same samples ${ }^{7}$.

The evolution of the densification ratio $\Delta \rho / \rho$ of a pure silica glass with $\mathrm{P}_{\max }$ is today well established in the case of a hydrostatic compression at ambient temperature ${ }^{5,7}$. This evolution is well described by the following sigmoidal relation:

$$
\frac{\Delta \rho}{\rho}=\left[\frac{1}{1+\beta \exp \left[-P_{\max } / P_{0}\right]}\right]\left(\frac{\Delta \rho}{\rho}\right)_{\max }
$$

where $(\Delta \rho / \rho)_{\max }=21 \%$ is the maximum densification ratio, $\beta=$ 5000 and $\mathrm{P}_{0}=1.67 \mathrm{GPa}$ are two fitting parameters.

According to Eq. 1, the evolution of the longitudinal sound velocity $c_{1}$ as a function of the densification ratio $\Delta \rho / \rho$ can be deduced from the Brillouin shift $v_{1}$ and the refractive index $n$, which both depend on $P_{\max }$, i.e on the densification ratio (see Eq. 3). The dependence of $v_{1}$ is given by Eq. 2 and the variation of the refractive index $n$ has been deduced from the data of Tan et al. ${ }^{38}$, which have highlighted the linear relation linking $\mathrm{n}$ and $\Delta \rho / \rho$ in permanently densified silica:

$$
n=n_{0}+\left[\frac{n_{\max }-n_{0}}{(\Delta \rho / \rho)_{\max }}\right]\left(\frac{\Delta \rho}{\rho}\right)
$$

where $\mathrm{n}_{0}=1.459$ and $\mathrm{n}_{\max }=1.543$ are respectively the refractive index of non-densified and fully-densified (21\%) silica glass. Hence, combining the four previous equations, the evolution of the longitudinal sound velocity as a function of the densification ratio has been calculated and is displayed in Fig. $2 \mathrm{~b}$.

The longitudinal sound velocity $\mathrm{c}_{1}$ decreases from $5930 \mathrm{~m} . \mathrm{s}^{-1}$ to $5840 \mathrm{~m} . \mathrm{s}^{-1}$ when increasing $\Delta \rho / \rho$ from 0 to $5 \%$. Above $5 \%, \mathrm{c}_{\mathrm{l}}$ increases monotonously with the densification up to $7050 \mathrm{~m} \cdot \mathrm{s}^{-1}$. Thus, the longitudinal sound velocity exhibits a minimum as a function of the densification ratio. Such behaviour recalls the well-known compressibility anomaly observed in silica glass under pressure, between $\mathrm{P}_{\text {atmo }}$ and $3 \mathrm{GPa}^{39-48}$. Nevertheless, these two anomalies need to be clearly distinguished: one appears after plastic deformation while the other takes place in the elastic regime of silica glass. This aspect will be discussed later. To go further, we have evaluated the elastic moduli of permanently densified silica glasses. The longitudinal (or P-waves) modulus $\mathrm{L}$, function of the density and longitudinal sound velocity $c_{l}$, can be directly deduced from our Brillouin scattering measurements and is reported in Fig. 3. The analytical relation linking $\mathrm{L}$ to $\Delta \rho / \rho$ can be deduced from the four previous equations.

In the range $\Delta \rho / \rho=[0-0.21]$, this relation between $\mathrm{L}$ and $\Delta \rho / \rho$ is accurately fitted by a second order polynomial function:

$$
L=L_{0}+L_{1} \frac{\Delta \rho}{\rho}+L_{2}\left(\frac{\Delta \rho}{\rho}\right)^{2}
$$

where $\mathrm{L}_{0}=77 \mathrm{GPa}$ is the longitudinal modulus of pristine silica glass, $\mathrm{L}_{1}=-24 \mathrm{GPa}$ and $\mathrm{L}_{2}=1370 \mathrm{GPa}$.

On the other hand, the transverse sound velocity, which would provide all the other elastic moduli, is lacking. However, for isotropic

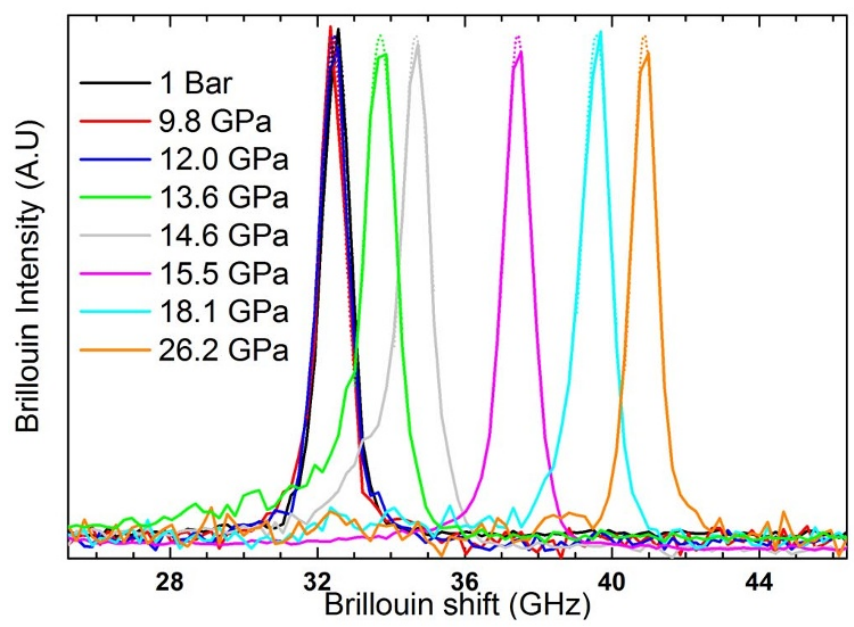

Figure 1 Brillouin spectra of the pristine silica sample (black line) and of the permanently densified a- $\mathrm{SiO}_{2}$ samples after hydrostatic compression in DAC (the spectra are recorded ex situ). The first three Brillouin lines (1 Bar, 9.8 and 12.0 GPa) are almost indistinguishable. Dotted lines represent the Lorentzian fits. 

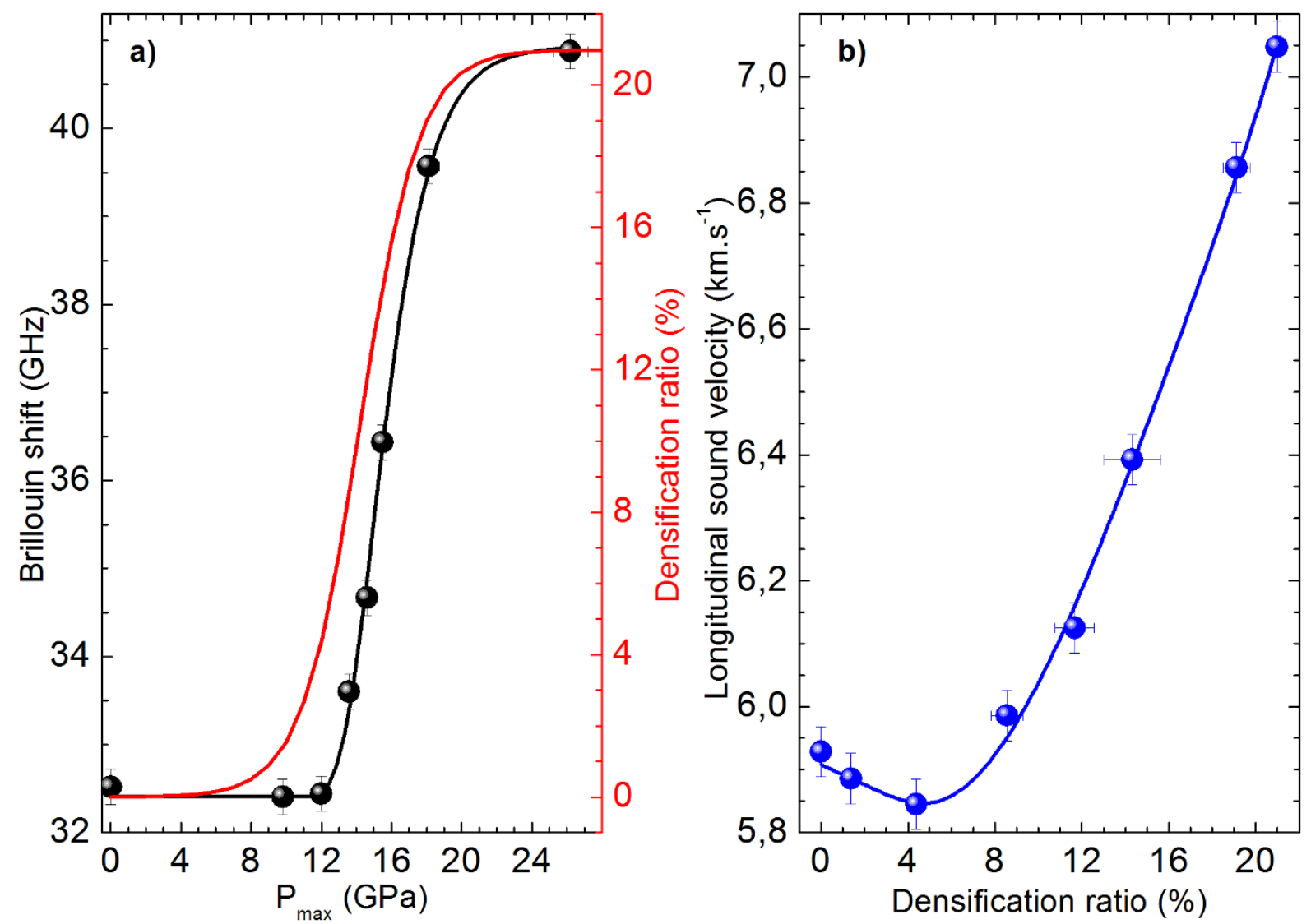

Figure $2 \mid$ (a) Longitudinal Brillouin line shift versus pressure reached $\mathrm{P}_{\max }$, recorded at atmospheric pressure, i.e after densification. The experimental points (black circles) are fitted using a Gompertz function (black line). The red line represents the evolution of the permanent densification ratio (right scale) as a function of $\mathrm{P}_{\max }{ }^{5-7}$. (b) Longitudinal wave velocity $c_{1}$ as a function of permanent densification ratio, calculated by combining Eq. 1, 2, 3 and 4. $c_{1}$ displays a minimum at $\Delta \rho / \rho=5 \%$.

solids such as glasses, the generalized Cauchy relation ${ }^{49}$, linking the longitudinal modulus $\mathrm{L}$ to the shear modulus $\mathrm{G}$, can be applied:

$$
L=a+b G
$$

This relation was shown to hold with constant values $\mathrm{a}=-24.8$ and $b=3.36$ for differently densified silica glass samples ${ }^{18}$.

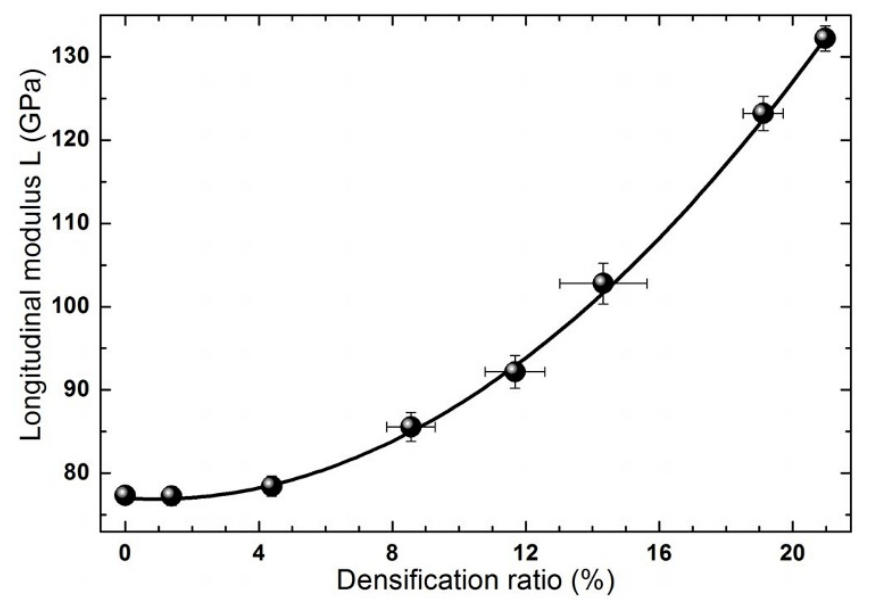

Figure 3 Longitudinal modulus L of silica glass as a function of the densification ratio. Full black circles are the data calculated from Brillouin scattering results. The black line represents the polynomial fit from Eq. 5.
Knowing $\mathrm{L}$ and $\mathrm{G}$ as a function of the permanent densification ratio, all the elastic moduli can be then determined, applying the well-known relations for homogeneous isotropic materials recalled in Table 2.

The evolution of the shear, bulk, and Young moduli (as well as the Poisson ratio) with densification ratio are displayed in Fig. 4.

The L, G, K and E moduli exhibit similar behaviours with densification: a monotonous enhancement is observed from 0 to $21 \%$ densification ratio, but the rate of this enhancement is extremely low, up to $\Delta \rho / \rho=5 \%$, due to the softening of the sound velocity in this densification range. The bulk modulus shows the greater enhancement, from $37 \mathrm{GPa}$ for a non-densified $\mathrm{SiO}_{2}$ glass to $70 \mathrm{GPa}$ for a fully densified one. Although the existing data concerning elastic moduli after cold densification of silica glass are sparse in the literature, some comparisons with previous experiments can be made. From the longitudinal and transverse sound velocities measurements of Polian et $a l .{ }^{40}$ and Zha et $a l^{41}$ for pristine and fully densified silica glass in a $\mathrm{DAC}$, the deduced elastic moduli $\mathrm{L} \approx$ $77 \mathrm{GPa}, \mathrm{G} \approx 32 \mathrm{GPa}, \mathrm{K} \approx 34 \mathrm{GPa}, \mathrm{E} \approx 73 \mathrm{GPa}$ and $\mathrm{L} \approx$ $129 \mathrm{GPa}, \mathrm{G} \approx 46 \mathrm{GPa}, \mathrm{K} \approx 67 \mathrm{GPa}, \mathrm{E} \approx 112 \mathrm{GPa}$ respectively are in excellent agreement with our results. As shown in Fig. $4 \mathrm{~d}$, the

Table 2 | Relations between elastic moduli: $K, v$, and $E$ as a function of $L$ and $G$

\begin{tabular}{ccc}
$K$ & $v$ & $E$ \\
\hline$L-\frac{4}{3} G$ & $\frac{L-2 G}{2 L-2 G}$ & $G\left(\frac{3 L-4 G}{L-G}\right)$
\end{tabular}



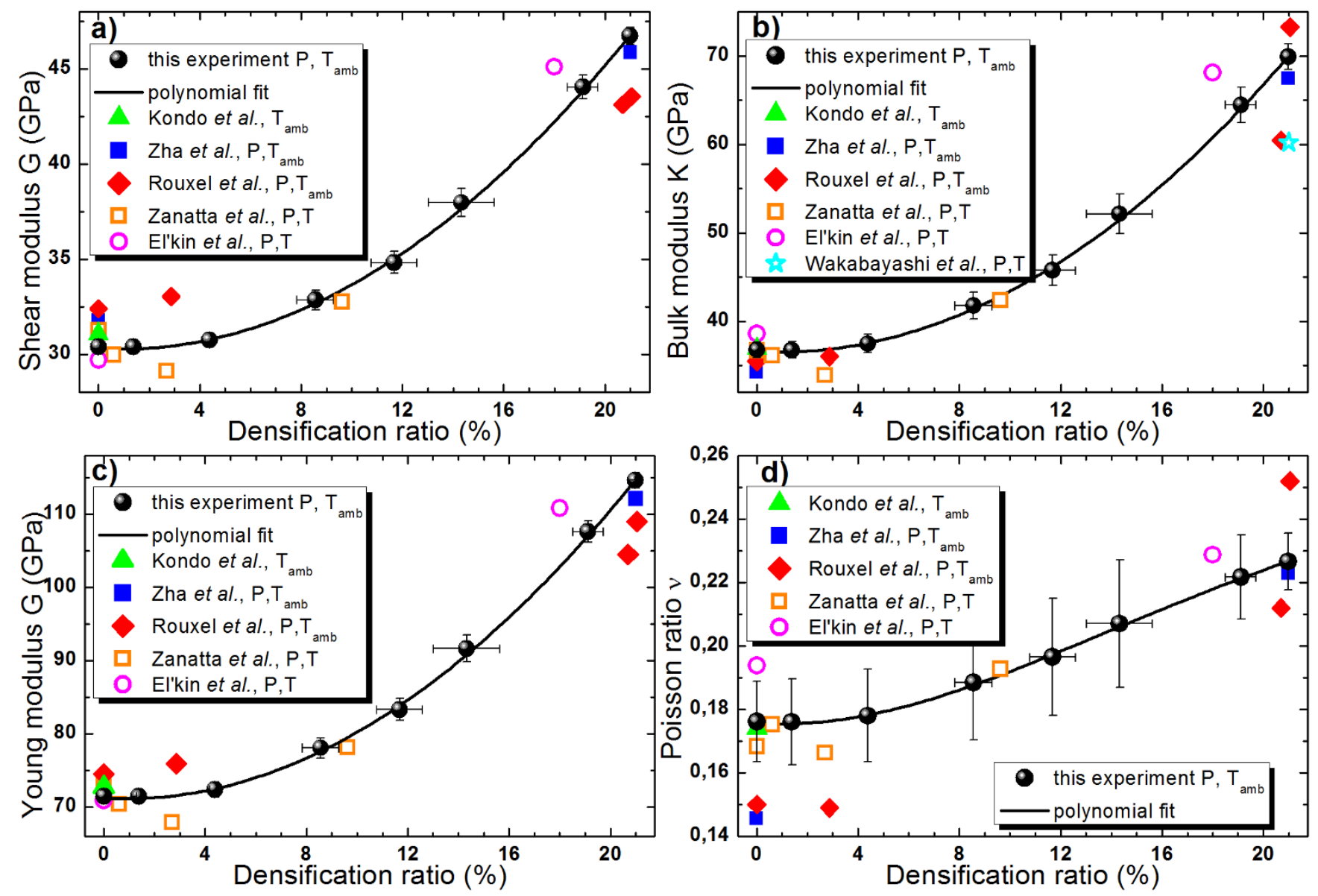

Figure $4 \mid$ (a) Shear modulus G, (b) Bulk modulus K, (c) Young modulus E and (d) Poisson ratio $v$ as a function of the densification ratio. Full black circles are deduced from our Brillouin scattering measurements, applying the Cauchy relation Eq.6. The lines represents the fits deduced from Eq. 5,6 and Table 2. The results are compared with previous measurements after densification at $\mathrm{T}_{\mathrm{amb}}$ by Kondo et al..$^{50}$ (full green triangles), Zha et al. ${ }^{41}$

(full blue squares) and Rouxel et al. ${ }^{3}$ (full red diamonds), and after densification at high temperature by Zanatta et al. ${ }^{18}$ (empty orange squares), El'kin et al. ${ }^{51}$ (empty purple circles) and Wakabayashi et al. ${ }^{19}$ (empty cyan star).

evolution of the Poisson ratio is roughly constant up to $5 \%$ densification $(v \approx 0.18)$, and then increases almost linearly to $v \approx 0.23$ up to a densification ratio of $21 \%$. These data are also in good agreement with previous measurements on permanently densified samples at $\mathrm{T}_{\mathrm{amb}}{ }^{3,35,41}$, thereby further validating the use of the Cauchy relation.

Let us now compare our results with elastic moduli of densified a$\mathrm{SiO}_{2}$ at high temperature, determined by Brillouin scattering ${ }^{18}$, ultrasonic measurements ${ }^{51}$ and by fitting the Birch-Murnaghan equation of states to the volume data recorded during compression ${ }^{19}$. For cold and hot compressions, the $\mathrm{P}$-T routes to plastically deform $\mathrm{SiO}_{2}$ glass are extremely different. Such differences in thermodynamic paths provide glasses with same density but different structures ${ }^{52}$. Despite this, the elastic moduli are similar (Fig. 4). This result supports the conclusion that the values of the elastic moduli only depend on the densification ratio of the glass, and not on the thermodynamical way to reach the permanent densification. This means that the relations given previously linking elastic moduli with $\Delta \rho / \rho$ are valuable for all densified $\mathrm{a}-\mathrm{SiO}_{2}$, whatever the thermodynamic paths taken to compress them.

\section{Discussion}

In order to discuss the permanent structural changes responsible for such evolution of the elastic moduli, first let us recall some general aspects concerning the densification process. At ambient pressure, silica glass has an open three-dimensional network structure, consisting of corner-shared $\mathrm{SiO}_{4}$ tetrahedra which forms wide $\mathrm{Si}-\mathrm{O}-\mathrm{Si}$ bond-angles and ring size distributions. Several kinds of structural transformations occur during the compaction under high pressure. Between $\mathrm{P}_{\mathrm{atmo}}$ and $3 \mathrm{GPa}$, reversible structural transitions similar to those which underlie the $\beta \rightarrow \alpha$ transition in cristobalite, first suggested by Vukcevich ${ }^{36}$, were recently observed in molecular-dynamic simulations ${ }^{44,45}$. This change in the six-fold ring conformation is responsible for the softening of the elastic moduli under pressure, i.e the well-known "compressibility anomaly" ${ }^{39-43}$. Indeed, the $\beta$-like rings, which are more symmetric than $\alpha$-like rings, have higher elastic moduli. Upon compression, the $\beta$-like rings collapse into $\alpha$ like rings by an abrupt rotation of $\mathrm{Si}-\mathrm{O}-\mathrm{Si}$ bridges, and the elastic moduli of silica glass decrease gradually from $\mathrm{P}_{\text {atmo }}$ up to $3 \mathrm{GPa}$. A gradual reduction of the $\mathrm{Si}-\mathrm{O}-\mathrm{Si}$ bond angle is also observed under compaction and is reversible below $9 \mathrm{GPa}$. The rate of the $\mathrm{Si}-\mathrm{O}-\mathrm{Si}$ angle reduction is maximal at $3 \mathrm{GPa}$, i.e at the pressure where the compressibility is maximal ${ }^{42}$. Below $9 \mathrm{GPa}$, the structural transformation occurs without breaking any bonds.

Above $9 \mathrm{GPa}$ which marks the elastic-plastic transition, the glass deformation results in an irreversible change of the network topology implying permanent densification. The appearance of intermediate fivefold Si-O coordination defects explains the network reorganization $^{23,28,46}$. These local plastic events are gradual between 9 and $25 \mathrm{GPa}$, as can be seen on previous Raman scattering experiments ${ }^{7}$. The intermediate range order is highly affected by the densification process. Notably the ring statistics change drastically, with the formation of smaller average ring size ${ }^{55}$, and the $\mathrm{Si}-\mathrm{O}-\mathrm{Si}$ angles gradually decrease with permanent densification ${ }^{7,26}$. These structural transformations are responsible for the permanent densification, 

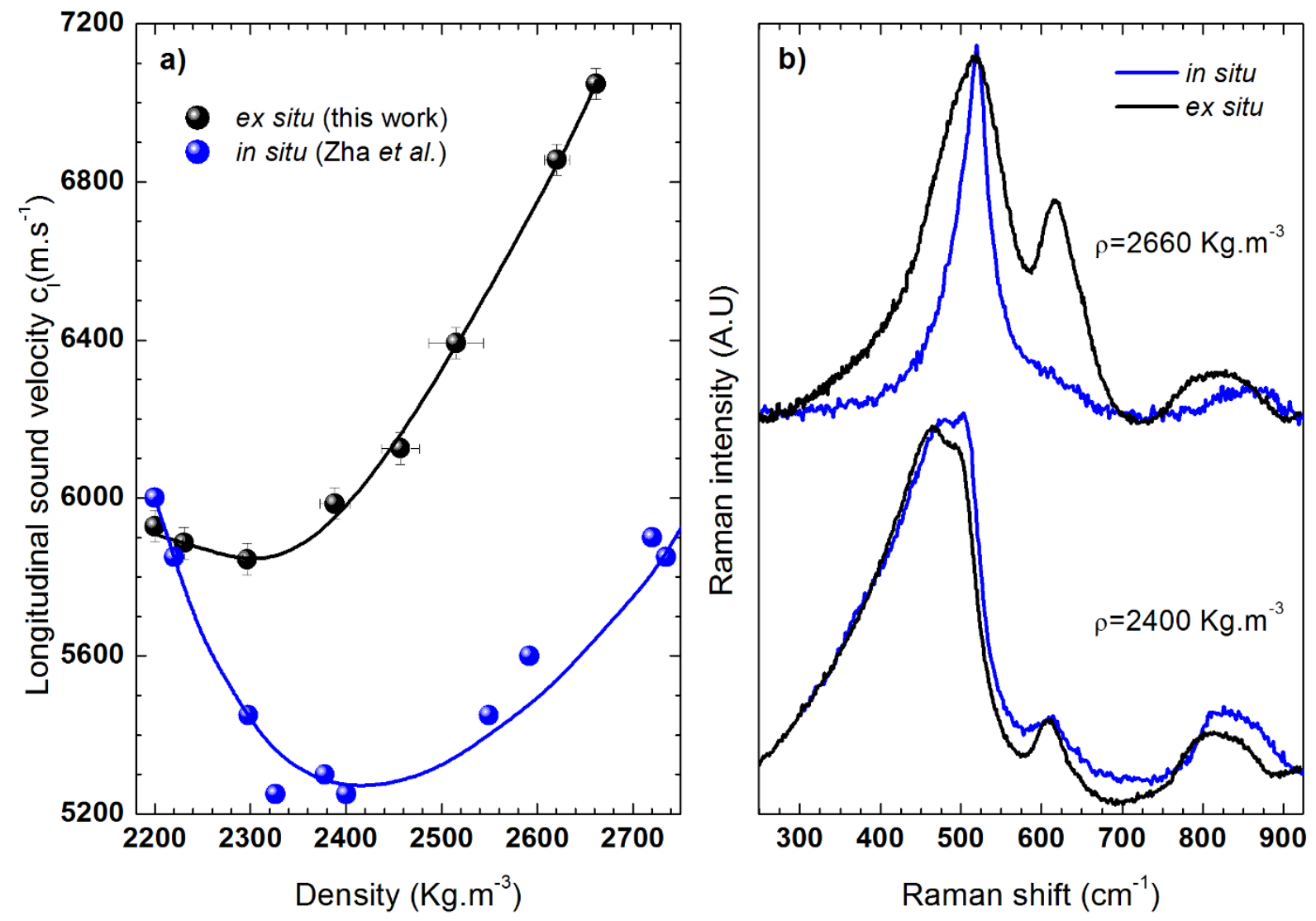

Figure $\mathbf{5} \mid$ (a) Longitudinal sound velocity $c_{1}$ as a function of density on permanently densified silica glass (black circles) and during in situ experiments (blue circles from Zha et al. ${ }^{41}$ ). (b) Comparison of Raman spectra recorded in situ (blue lines) and ex situ (black lines) on glasses of same density, at two different densities $\rho=2400 \mathrm{Kg} \cdot \mathrm{m}^{-3}$ and $\rho=2660 \mathrm{Kg} . \mathrm{m}^{-3}$. The ex situ spectra are extracted from Deschamps et al. ${ }^{7}$ and in situ spectra have been recorded at 3.0 GPa and $6.7 \mathrm{GPa}$, corresponding respectively to a density of $\rho=2400 \mathrm{Kg} \cdot \mathrm{m}^{-3}$ and $\rho=2660 \mathrm{Kg} \cdot \mathrm{m}^{-3}$ during elastic deformation.

which saturates for a compression-decompression cycle up to $25 \mathrm{GPa}$, and were previously interpreted in terms of a growing proportion of high density amorphous (HDA) local structure ${ }^{48}$ in the frame of the LDA-HDA amorphous-amorphous transition ${ }^{15,53,54}$. When $P_{\max } \geq 25 \mathrm{GPa}$, the maximum densification ratio of $21 \%$ is reached, indicating the inability of silica to accommodate further irreversible structural changes. Further compression of silica glass above $20-25 \mathrm{GPa}$ leads to an increase in the $\mathrm{Si}-\mathrm{O}$ coordination number, from a tetrahedral to an octahedral amorphous network ${ }^{55,56}$. This reversible transformation ends around $40 \mathrm{GPa}^{57,58}$.

Using Brillouin spectroscopy, we have shown herein that after cold compression the longitudinal wave velocity decreases with the densification ratio at the beginning of the permanent densification. Such behaviour was also reported recently on $\mathrm{SiO}_{2}$ samples heated and quenched at high pressure in a multi-anvil apparatus ${ }^{18}$. In this later article, the authors explain that the resulting sound velocities are consistent with previous measurements of Zha et al. ${ }^{41}$. However, the measurements of Zha et al. concern the elastic deformation of silica glass followed in situ by Brillouin scattering. We argue that such comparison between plastic and elastic deformations appears to be incorrect. To illustrate this point, we plot Fig. 5a the longitudinal sound velocity as a function of silica glass density from in situ experiments ${ }^{41}$ and ex situ experiments (our data and the one of Zanatta et al. ${ }^{18}$ ). It is worth noting that the article of Zha et al. displays sound velocity as a function of pressure, but also provides the relation between the applied hydrostatic pressure and the in situ glass density.

For both ex situ and in situ experiments, the longitudinal sound velocity $c_{1}$ displays a non-monotonous behaviour, with a minimum as a function of the density. However, the two behaviours must be distinguished. For in situ measurements, $c_{1}$ decreases strongly from
$6000 \mathrm{~m} \cdot \mathrm{s}^{-1}$ at $\mathrm{P}_{\text {atmo }}\left(\rho=2200 \mathrm{Kg} \cdot \mathrm{m}^{-3}\right)$ down to $5300 \mathrm{~m} \cdot \mathrm{s}^{-1}$ at $3 \mathrm{GPa}\left(\rho=2400 \mathrm{Kg} \cdot \mathrm{m}^{-3}\right)$, and then increases monotonously. We note that $c_{1}=5600 \mathrm{~m} \cdot \mathrm{s}^{-1}$ at $6.7 \mathrm{GPa}\left(\rho=2660 \mathrm{Kg} \cdot \mathrm{m}^{-3}\right)$. The large amplitude of the anomalous decrease of $c_{1}$ observed in situ at the beginning of the compression makes the anomaly visible on the elastic moduli.

This behaviour is at variance with ex situ experiments, where $c_{1}$ first decreases slightly from $6000 \mathrm{~m} . \mathrm{s}^{-1}$ to $5850 \mathrm{~m} \cdot \mathrm{s}^{-1}$ at $\rho=$ $2300 \mathrm{Kg} \cdot \mathrm{m}^{-3}$ and then increases drastically up to $7050 \mathrm{~m} \cdot \mathrm{s}^{-1}$ at the density maximum of a permanently compressed silica glass $(\rho=$ $2660 \mathrm{Kg} \cdot \mathrm{m}^{-3}$ ). The longitudinal sound velocity experiences a much less pronounced minimum than the one observed in situ, and the elastic moduli display a monotonous behaviour with densification. At $\rho=2660 \mathrm{Kg} \cdot \mathrm{m}^{-3}, \mathrm{c}_{1}$ is largely above the initial value, implying the hardening of the glass with densification (Fig. 3), while at this density, in situ data show that $\mathrm{c}_{\mathrm{l}}<6000 \mathrm{~m} . \mathrm{s}^{-1}$.

In order to compare the structure of silica glass at similar densities during in situ experiments and after permanent densification, Fig. 5b displays simultaneously in situ and ex situ Raman spectra at $\rho=$ $2400 \mathrm{Kg} \cdot \mathrm{m}^{-3}$ and $\rho=2660 \mathrm{Kg} \cdot \mathrm{m}^{-3}$. The ex situ spectra have been recorded on the samples recovered after compression in the DAC up to $\mathrm{P}_{\max }=13.6 \mathrm{GPa}$ and $26.2 \mathrm{GPa}$, i.e densified at $\rho=2400 \mathrm{Kg} \cdot \mathrm{m}^{-3}$ and $\rho=2660 \mathrm{Kg} \cdot \mathrm{m}^{-3}$ respectively. The in situ spectra have been recorded in the $\mathrm{DAC}$ at $\mathrm{P}=3 \mathrm{GPa}$ and $\mathrm{P}=6.7 \mathrm{GPa}$, corresponding to those same densities ${ }^{41}$. These spectra clearly confirm the fundamental differences between in situ and ex situ experiments. At equivalent density, strong discrepancies appear in the $\mathrm{a}-\mathrm{SiO}_{2}$ structure. In particular, the main band is sharper and the $\mathrm{D}_{2}$ band (breathing modes of 3-membered rings) located at $600 \mathrm{~cm}^{-1}$ is weaker in situ, revealing strong differences in the $\mathrm{Si}-\mathrm{O}-\mathrm{Si}$ angles distribution 
and ring statistics. The planar geometry and the high symmetry of small rings conferring a lower compressibility to $\mathrm{a}-\mathrm{SiO}_{2}$ network, the formation of smaller rings during plastic deformation is probably responsible for the glass hardening. Such permanent structural transformations have also been revealed by Raman spectroscopy on pure silica glass after indentation ${ }^{59,60}$. Indeed, indentation causes an increase of the $\mathrm{D}_{1}$ and $\mathrm{D}_{2}$ defect lines and a shift of the main band to higher frequencies. The similarities observed when comparing the $\mathrm{SiO}_{2}$ Raman spectra after hydrostatic compression and indentation clearly shows that the plastic deformation through indentation is driven by compaction rather than shear-flow. More details concerning ex situ Raman characterization and its relevance to characterize indented glasses can be found in a previous study (see Deschamps et al. ${ }^{7}$ ).

In spite of the fundamental differences between in situ and ex situ transformations, the decrease of the sound velocity as a function of the density observed at the beginning of the permanent densification can be still explained by the $\beta \rightarrow \alpha$ cristobalite-like transition which gradually softens the structure. Up to the elastic yield around $9 \mathrm{GPa}$, this transition is fully reversible. Above $9 \mathrm{GPa}$, the plastic deformation probably involves a gradual and permanent conformation change from $\beta$-like to $\alpha$-like 6 -fold rings: a certain part (depending on $\mathrm{P}_{\max }$ ) of the $\beta$ rings population is permanently transformed into $\alpha$ rings type. Such phenomenon perfectly describe the gradual weakening and loss of the compressibility anomaly with the densification ratio $^{47,48}$. The minimum of the sound velocity versus permanent densification can be well explained by the competition between two different irreversible mechanisms: the $\beta \rightarrow \alpha$ transition which softens the structure, and the formation of smaller rings making the network elastically stronger.

In the recent article of Keryvin et al. ${ }^{35}$ which proposes a mechanical model of silica glass under hydrostatic pressure, the authors use a linear function to describe the evolution of the elastic moduli versus densification ratio. It is also specified by the authors that the lack of information between $4 \%$ and $20 \%$ prevents a more accurate law. Our results clearly reveal that the evolution of elastic moduli is far from linear (see Fig. 3 and 4). Taking into account the polynomial laws proposed previously would considerably improve such modelling, whether for hydrostatic compression or indentation ${ }^{31,35}$.

\section{Methods}

A diamond anvil cell (DAC) was used to densify Suprasil 300 vitreous $\mathrm{SiO}_{2}$ from Heraeus $([\mathrm{OH}<1 \mathrm{ppm}]$ at ambient temperature. The pressures reached in the DAC were determined in situ by the ruby-fluorescence method. The hole of the metal gasket, $200 \mu \mathrm{m}$ in diameter, was filled with a glass splinter (typically $50 \mu \mathrm{m}$ in size), several ruby spheres, and a liquid pressure medium (a $20: 4: 1$ mixture of methanolethanol-water). This pressure medium has been reported to remain perfectly hydrostatic up to $12 \mathrm{GPa}$ and quasi-hydrostatic above ${ }^{37}$. All the recovered samples were in one piece, suggesting low shear stresses during the compression-decompression cycle.

The Brillouin scattering signal was recorded using a Sandercock tandem Fabry Perot coupled with a microscope in back-scattering geometry. The excitation source used was a $\mathrm{Nd}^{3+}$-YAG laser operating at $\lambda=532 \mathrm{~nm}$.

1. Taylor, E. W. Plastic deformation of optical glass. Nature 163, 323-323 (1949).

2. Bridgman, P. W. \& Simon, I. Effects of very high pressures on glass. J. Appl. Phys. 24, 405-413 (1953).

3. Rouxel, T., Ji, H., Guin, J. P., Augereau, F. \& Rufflé, B. Indentation deformation mechanism in glass: Densification versus shear flow. J. Appl. Phys. 107, 094903 (2010).

4. Deschamps, T., Martinet, C., Bruneel, J. L. \& Champagnon. Soda-lime silicate glass under hydrostatic pressure and indentation: A micro-Raman study. B. J. Phys.: Condens. Matter 23, 035402 (2011)

5. Rouxel, T., Ji, H., Hammouda, T. \& Moréac, A. Poisson's ratio and the densification of glass under high pressure. Phys. Rev. Lett. 100, 225501 (2008).

6. Greaves, G. N., Greer, A. L., Lakes, R. S. \& Rouxel, T. Poisson's ratio and modern materials. Nat. Mater. 10, 823-837 (2011).

7. Deschamps, T. et al. Permanent densification of compressed silica glass: a Ramandensity calibration curve. J. Phys.: Condens. Matter 25, 025402 (2013).

8. Mackenzie, J. D. \& Laforce, R. P. High-pressure densification of glass and the effects of shear. Nature 197, 480-481 (1963).
9. McMillan, P., Piriou, B. \& Couty, R. A Raman study of pressure-densified vitreous silica. J. Chem. Phys. 81, 4234-4236 (1984).

10. Polian, A. \& Grimsditch, M. Room temperature densification of a- $\mathrm{SiO}_{2}$ versus pressure. Phys. Rev. B 41, 6086-6087 (1990).

11. Grimsditch, M. Polymorphism in amorphous $\mathrm{SiO}_{2}$. Phys. Rev. Lett. 52, 2379-2381 (1984).

12. Sugai, S. \& Onodera, A. Medium-range order in permanently densified $\mathrm{SiO}_{2}$ and $\mathrm{GeO}_{2}$ glass. Phys. Rev. Lett. 77, 4210-4213 (1996).

13. Polsky, C., Smith, K. H. \& Wolf, G. H. Effect of pressure on the absolute Raman scattering cross section of $\mathrm{SiO}_{2}$ and $\mathrm{GeO}_{2}$ glasses. J. Non-Cryst. Solids 248, 159-168 (1999).

14. Schroeder, J. et al. Raman scattering and Boson peaks in glasses: Temperature and pressure effects. J. Non-Cryst. Solids 349, 88-97 (2004).

15. Champagnon, B. et al. High pressure elastic and plastic deformations: in situ diamond anvil cell Raman experiments. J. Non-Cryst. Solids 354, 569-573 (2008).

16. Vandembroucq, D. et al. Density hardening plasticity and mechanical ageing of silica glass under pressure: a Raman spectroscopic study. J. Phys.: Condens. Matter 20, 485221 (2008).

17. Poe, B. T., Romano, C. \& Henderson, G. Raman and XANES spectrocopy of permanently densified vitreous silica. J. Non-Cryst. Solids 341, 162-169 (2004).

18. Zanatta, M. et al. Elastic properties of permanently densified silica glass: a Raman, Brillouin light, and x-ray scattering study. Phys. Rev. B 81, 212201 (2010).

19. Wakabayashi, D., Funamori, N., Sato, T. \& Taniguchi, T. Compression behaviour of densified $\mathrm{SiO}_{2}$ glass. Phys. Rev. B 84, 144103 (2011).

20. Wu, M., Liang, Y., Jiang, J. Z. \& John, S. T. Structure and properties of dense silica glass. Sci. Rep. 2, 398 (2012).

21. Yuan, F. \& Huang, L. Brittle to ductile transition in densified silica glass. Sci. Rep.4, 5035 (2014).

22. Jin, W., Kalia, R. K., Vashishta, P. \& Rino, J. P. Structural transformation in densified silica glass: a molecular-dynamics study. Phys. Rev. B 50, 118 (1994).

23. Della Valle, R. G. \& Venuti, E. High-pressure densification of silica glass: a molecular-dynamics simulation. Phys. Rev. B 54, 3809 (1996).

24. Lacks, D. J. First order amorphous-amorphous transformation in silica. Phys. Rev. Lett. 84, 4629-4632 (2000).

25. Trachenko, K. \& Dove, M. T. Intermediate states in pressurized silica glass: Reversibility window analogue. Phys. Rev. B 67, 212203 (2003).

26. Huang, L. \& Kieffer, J. Amorphous-amorphous transitions in silica glass. II. Irreversible transitions and densification limit. Phys. Rev. B 69, 224204 (2004).

27. Léonforte, F., Tanguy, A., Wittmer, J. P. \& Barrat, J. L. Inhomogeneous elastic response of silica glass. Phys. Rev. Lett. 97, 055501 (2006).

28. Liang, Y., Miranda, C. R. \& Scandolo, S. Mechanical strength and coordination defects in compressed silica glass: Molecular-dynamics simulations. Phys. Rev. B 75, 024205 (2007).

29. Izvekov, S. \& Rice, B. M. Mechanism of densification in silica glass under pressure as revealed by a bottom-up pairwise effective interaction model. J. Chem. Phys. 136, 134508 (2012)

30. Li, N., Sakidja, R., Aryal, S. \& Ching, W. Y. Densification of a continuous random network model of amorphous $\mathrm{SiO}_{2}$ glass. Phys. Chem. Chem. Phys. 16, 1500-1514 (2014).

31. Kermouche, G., Barthel, E., Vandembroucq, D. \& Dubujet, P. Mechanical modelling of indentation-induced densification in amorphous silica. Acta Mater. 56, 3222-3228 (2008).

32. Gadelrab, K. R., Bonilla, F. A. \& Chiesa, M. Densification modelling of fused silica under nanoindentation. J. Non-Cryst. Solids 358, 392-398 (2012).

33. Lacroix, L., Teisseire, J., Kermouche, G. \& Barthel, E. Plastic deformation and residual stresses in amorphous silica pillars under uniaxial loading. Acta Mater. 20, 5555-5566 (2012)

34. Kylimis, D. A. \& Delaye, J. M. Nanoindentation of pristine and disordered silica: Molecular-dynamics simulation. J. Non-Cryst. Solids 382, 87-94 (2013).

35. Keryvin, V. et al. Constitutive modeling of the densification process in silica glass under hydrostatic pressure. Acta Mater. 62, 250 (2014).

36. Vukcevich, M. R. A new interpretation of the anomalous properties of vitreous silica. J. Non-Cryst. Solids 11, 25-63 (1972).

37. Klotz, S., Chervin, J. C., Munsch, P. \& Le Marchand, G. J. Hydrostatic limits of 11 pressure transmitting media. Phys. D: Appl. Phys. 42, 075413 (2009).

38. Tan, C., Arndt, J. \& Xie, H. Optical properties of densified silica glass. Physica B 252, 28-33 (1998).

39. Meade, C. \& Jeanloz, R. Frequency-dependent equation of state of fused silica to 10 GPa. Phys. Rev. B 35, 236-244 (1987).

40. Polian, A. \& Grimsditch, M. Sound velocities and refractive index of densified a$\mathrm{SiO}_{2}$ to $25 \mathrm{GPa}$. Phys. Rev. B 47, 13979-13982 (1993).

41. Zha, C. S., Hemley, R. J., Mao, H. K., Duffy, T. S. \& Meade, C. Sound velocities and refractive index of $\mathrm{SiO}_{2}$ glass to $57.5 \mathrm{GPa}$ by Brillouin scattering. Phys. Rev. B 50, 13105-13112 (1994).

42. Deschamps, T., Martinet, C., de Ligny, D. \& Champagnon, B. Elastic anomalous behaviour of silica glass under high-pressure: In situ Raman study. J. Non-Cryst. Solids 355, 1095-1098 (2009).

43. Deschamps, T., Martinet, C., Bruneel, J. L., de Ligny, D. \& Champagnon, B. Lowfrequency Raman scattering under high pressure in diamond anvil cell: Experimental protocol and application to $\mathrm{GeO} 2$ and $\mathrm{SiO} 2$ boson peaks. J. NonCryst. Solids 358, 3156-3160 (2012). 
44. Huang, L. \& Kieffer, J. Amorphous-amorphous transitions in silica glass. I. Reversible transitions and thermomechanical anomalies. Phys. Rev. B 69, 224203 (2004).

45. Huang, L., Duranduru, M. \& Kieffer, J. Transformation pathways of silica under high pressure. Nat. Mater. 5, 977-981 (2006)

46. Mantisi, B., Tanguy, A., Kermouche, G. \& Barthel, E. Atomistic response of a model silica glass under shear and pressure. Eur. Phys. J. B 85, 304 (2012).

47. Huang, L. \& Kieffer, J. Anomalous thermomechanical properties and laser induced densification of vitreous silica. Appl. Phys. Lett. 89, 141915 (2006).

48. Sonneville, S. et al. Progressive transformations of silica glass upon densification. J. Chem. Phys. 137, 124505 (2012).

49. Wang, W. H. The elastic properties, elastic models and elastic perspectives of metallic glasses. Prog. Mater. Sci. 57, 487-656 (2012)

50. Kondo, K., Ito, S. \& Sawaoka, A. Nonlinear pressure dependence of the elastic moduli fused quartz up to $3 \mathrm{GPa}$. J. Appl. Phys. 52, 2826-2831 (1981).

51. El'kin, F. S., Brazhkin, V. V., Khvostantsev, L. G., Tsiok, O. B. \& Lyapin, A. G. In situ study of the mechanism of formation of pressure-densified $\mathrm{SiO}_{2}$ glasses. JETP Lett. 75, 342-347 (2002).

52. Liang, Y., Miranda, C. R. \& Scandolo, S. Temperature-induced densification of compressed $\mathrm{SiO}_{2}$ glass: A molecular dynamics study. High Press. Res. 28, 35-44 (2008).

53. Brazhkin, V. V. \& Lyapin, A. G. High-pressure phase transformations in liquids and amorphous solids. J. Phys.: Condens. Matter 15, 6059-6084 (2003).

54. Furhmann, S., Deschamps, T., Champagnon, B. \& Wondraczek, L. A reconstructive polyamorphous transition in borosilicate glass induced by irreversible compaction. J. Chem. Phys. 140, 054501 (2014).

55. Meade, C., Hemley, R. J. \& Mao, H. K. High-pressure X-ray-diffraction of $\mathrm{SiO}_{2}$ glass. Phys. Rev. Lett. 69, 1387-1390 (1992)

56. Sato, T. \& Funamori, N. Sixfold-coordinated amorphous polymorph of $\mathrm{SiO} 2$ under high pressure. Phys. Rev. Lett. 101, 25502 (2008).

57. Benmore, C. J. et al. Structural and topological changes in silica glass at pressure. Phys. Rev. B 81, 054105 (2010).
58. Sato, T. \& Funamori, N. High-pressure structural transformation of $\mathrm{SiO}_{2}$ glass up to $100 \mathrm{GPa}$. Phys. Rev. B 82, 184102 (2010).

59. Perriot, A. et al. Raman microspectroscopic characterization of amorphous silica plastic behaviour. J. Am. Ceram. Soc. 89, 596-601 (2006).

60. Winterstein-Beckmann, A., Mönke, D., Palles, D., Kamitsos, E. I. \& Wondraczek, L. Raman spectroscopic study of structural changes induced by micro-indentation in low alkali borosilicate glasses. J. Non-Cryst. Solids 401, 110-114 (2014).

\section{Author contributions}

B.C. designed the research. T.D. and C.M. carried out high pressure experiments. J.M., T.D and A.M. carried out Brillouin measurements. T.D. performed data analysis and wrote the paper. All the authors reviewed the manuscript.

\section{Additional information}

Competing financial interests: The authors declare no competing financial interests.

How to cite this article: Deschamps, T., Margueritat, J., Martinet, C., Mermet, A. \& Champagnon, B. Elastic Moduli of Permanently Densified Silica Glasses. Sci. Rep. 4, 7193; DOI:10.1038/srep07193 (2014).

This work is licensed under a Creative Commons Attribution-NonCommercialShareAlike 4.0 International License. The images or other third party material in this article are included in the article's Creative Commons license, unless indicated otherwise in the credit line; if the material is not included under the Creative Commons license, users will need to obtain permission from the license holder in order to reproduce the material. To view a copy of this license, visit http:// creativecommons.org/licenses/by-nc-sa/4.0/ 